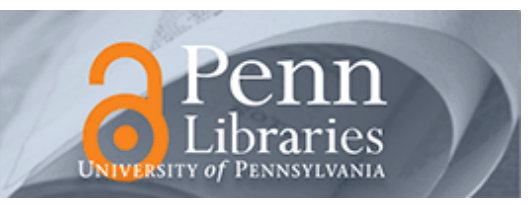

University of Pennsylvania

ScholarlyCommons

\title{
The Effect of an Adjacent Viscous Fluid on the Transmission of Torsional Stress Waves in a Submerged Waveguide
}

Jin O. Kim

University of Pennsylvania

Yuzhou Wang

University of Pennsylvania

Haim H. Bau

University of Pennsylvania, bau@seas.upenn.edu

Follow this and additional works at: https://repository.upenn.edu/meam_papers

Part of the Mechanical Engineering Commons

\section{Recommended Citation}

Kim, Jin O.; Wang, Yuzhou; and Bau, Haim H., "The Effect of an Adjacent Viscous Fluid on the Transmission of Torsional Stress Waves in a Submerged Waveguide" (1991). Departmental Papers (MEAM). 202.

https://repository.upenn.edu/meam_papers/202

Suggested Citation:

Kim, Jin O. Yuzhou Wang, Haim H. Bau. (1991) The effect of an adjacent viscous fluid on teh transmission of torsional stress waves in a submerged waveguide. Journal of the Acoustical Society of America. Vol 89(3). p.1414-1422.

Copyright 1991 American Institute of Physics. This article may be downloaded for personal use only. Any other use requires prior permission of the author and the American Institute of Physics.

The following article appeared in Journal of the Acoustical Society of America and may be found at http://scitation.aip.org/getabs/servlet/

GetabsServlet?prog=normal\&id=JASMAN000089000003001414000001\&idtype=cvips\&gifs=yes

This paper is posted at ScholarlyCommons. https://repository.upenn.edu/meam_papers/202

For more information, please contact repository@pobox.upenn.edu. 


\title{
The Effect of an Adjacent Viscous Fluid on the Transmission of Torsional Stress Waves in a Submerged Waveguide
}

\author{
Abstract \\ The effects of an adjacent fluid's viscosity and density on the characteristics of torsional stress waves \\ transmitted in a waveguide with a circular cross section are studied theoretically and experimentally. \\ Expressions for the torsional waves speed, dispersion relations, and attenuation are obtained as \\ functions of the adjacent fluid's viscosity and density. The theoretical results are compared with \\ experimental observations. It is demonstrated that a devices similar to the one described herein can be \\ used as a rugged, real-time, on-lines sensor for measuring the viscosity of a fluid with a known density. \\ Such a sensor can measure the viscosity of fluids with a density viscosity product $(\rho f \mu)$ greater than \\ $100 \mathrm{~kg}^{2} / \mathrm{m}^{4}$ s to a precision of $1 \%$ or better \\ Disciplines \\ Engineering | Mechanical Engineering

\section{Comments} \\ Suggested Citation: \\ Kim, Jin O. Yuzhou Wang, Haim H. Bau. (1991) The effect of an adjacent viscous fluid on teh transmission \\ of torsional stress waves in a submerged waveguide. Journal of the Acoustical Society of America. Vol \\ 89(3). p.1414-1422. \\ Copyright 1991 American Institute of Physics. This article may be downloaded for personal use only. Any \\ other use requires prior permission of the author and the American Institute of Physics. \\ The following article appeared in Journal of the Acoustical Society of America and may be found at \\ http://scitation.aip.org/getabs/servlet/ \\ GetabsServlet?prog=normal\&id=JASMAN000089000003001414000001\&idtype=cvips\&gifs=yes
}




\title{
The effect of an adjacent viscous fluid on the transmission of torsional stress waves in a submerged waveguide
}

\author{
Jin O. Kim, Yuzhou Wang, and Haim H. Bau \\ Department of Mechanical Engineering and Applied Mechanics, University of Pennsylvania, Philadelphia, \\ Pennsylvania 19104-6315
}

(Received 15 September 1989; accepted for publication 9 October 1990)

\begin{abstract}
The effects of an adjacent fluid's viscosity and density on the characteristics of torsional stress waves transmitted in a waveguide with a circular cross section are studied theoretically and experimentally. Expressions for the torsional wave speed, dispersion relations, and attenuation are obtained as functions of the adjacent fluid's viscosity and density. The theoretical results are compared with experimental observations. It is demonstrated that a device similar to the one described herein can be used as a rugged, real-time, on-line sensor for measuring the viscosity of a fluid with a known density. Such a sensor can measure the viscosity of fluids with a density viscosity product $\left(\rho_{f} \mu\right)$ greater than $100 \mathrm{~kg}^{2} / \mathrm{m}^{4} \mathrm{~s}$ to a precision of $1 \%$ or better.
\end{abstract}

PACS numbers: 43.85.Dj, 43.20.Mv, 43.20.Tb, 43.40.At

\section{INTRODUCTION}

The speed and attenuation of stress waves transmitted in submerged waveguides are affected by the viscosity and density of the surrounding fluid. When a stress wave travels in a solid, it causes deformation of the solid-fluid interface, which in turn induces fluid motion. The coupling mechanism between the motion in the solid and the fluid depends on the geometry of the waveguide and the type of stress wave employed. For example, when the solid's deformation is parallel to the solid-fluid interface, the coupling is solely due to viscous drag. When the deformation is normal to the surface, then the coupling results from a combination of both pressure and viscous drags. The former arises when shear waves are transmitted in a strip or torsional stress waves are transmitted in a circular cylinder, ' while the latter occurs when torsional stress waves are transmitted in waveguides with a noncircular cross section. ${ }^{2,3}$ Thus, by measuring the speed and the attenuation of stress waves in submerged solid waveguides, one can obtain information on various rheological properties of a fluid such as its viscosity and/or density.

The idea of correlating the rheological properties of fluids with the characteristics of stress waves transmitted in submerged solid waveguides is not new. Roth and Rich' suggested obtaining a fluid's viscosity by measuring the attenuation of shear waves transmitted in a strip waveguide submerged in fluids of known density. They advanced an approximate theory to correlate the speed and the attenuation of the stress wave with the fluid's viscosity density product $\left(\rho_{f} \mu\right)$ and employed their device to measure the viscosity of various viscoelastic fluids.

For many applications, it also may be desired to measure the liquid's density. To this end, Lynnworth ${ }^{2}$ employed torsional stress waves in waveguides with noncircular cross sections. In such situations, the fluid affects the stress waves primarily via "pressure drag." By transmitting torsional stress waves in waveguides with circular and noncircular cross sections, one can measure both fluid's viscosity and its density. ${ }^{4}$
This paper has been inspired by our attempts at measuring the viscosity of Newtonian liquids by transmitting torsional stress waves in submerged waveguides with circular cross sections. In order to achieve a deeper understanding of the sensor's operation as well as to obtain suggestions for possible improvernents of its sensitivity, we formulated a mathematical model somewhat more rigorous than the ones previously employed (i.e., Ref. 1). The model and the solution of the governing equations are presented in the first part of the paper. The exact solution of the equations leads to a transcendental, complex equation which allows one to calculate both the speed and attenuation of the stress wave. Since the exact solution consists of an implicit relation between the various quantities of interest, we also proceeded to develop a more convenient, explicit, asymptotic relation valid for relatively low viscosity fluids. A comparison of the exact and asymptotic solutions reveals that the asymptotic solution is useful for a wide range of applications. In the second part of the paper, we report a few experimental results and compare them with the theoretical predictions. Finally, we suggest various ways to improve the sensor's sensitivity. The results reported herein may also be useful for the design of delay lines.

Since viscosity measurement is important in a variety of industrial processes, it is not surprising that a considerable amount of effort has been invested in the development of various viscosity measurement devices. Due to space constraints, we will not review the entire field here. Rather we mention only the devices which are most closely related to the one described here. These devices involve observing the period and decay (damping) constant of torsional oscillations of an axially symmetric body, such as a disk, ${ }^{5,6}$ a cup, ${ }^{7,8}$ a sphere, ${ }^{9}$ or a cylinder, ${ }^{10}$ suspended from an elastic strand and submerged in the measured fluid. The device described in this paper is likely to be less accurate than the aforementioned devices but it has the advantage of being rugged, having no moving components, and enabling one to measure viscosity on line and in real time. These attributes may make such a device attractive to industrial users. 


\section{THE APPARATUS}

The apparatus consists of a waveguide, made of an elastic material of density $\rho_{s}$, with a uniform circular cross section, submerged in a fluid of density $\rho_{f}$ and viscosity $\mu$. The waveguide is subjected to a torsional pulse which is conveniently induced utilizing the magnetostrictive phenomenon. Briefly, one end of a delay line, made of a magnetostrictive material, is soldered or glued to the waveguide while a coil is placed around its other end, as shown in Fig. 1. The delay line is electrically polarized so as to develop a circumferential, permanent magnetic field inside the magnetostrictive wire. The introduction of a current pulse in a coil causes a time varying axial magnetic field to develop. The interaction between the two aforementioned magnetic fields leads to a twisting force on the magnetostrictive wire and the generation of a torsional pulse. This is known as the Wiedemann effect." The resulting torsional stress wave travels in the magnetostrictive wire. Part of the wave is reflected at the magnetostrictive wire-waveguide interface. The other part travels through the waveguide and is reflected from its other end. The reflected waves cause electromotive forces in the coil which now acts as a receiver (the inverse Wiedemann effect). The signal can be viewed on an oscilloscope's screen. By measuring the time which elapses between the two signals and their amplitudes, one can calculate the speed and attenuation of the torsional stress wave in a waveguide of known length. In our experiments, the time span is measured peak to peak with a precision of $5 \mathrm{~ns}$ using a digital oscilloscope. As we shall show later, this time span (or the wave speed) depends, among other things, on the waveguide's and the adjacent fluid's densities, on the fluid's viscosity, and on the waveguide's geometry.

In our experiments, we typically employ waveguides made of solid and hollow aluminum rods (density $\rho_{s}$ $=2.70 \times 10^{3} \mathrm{~kg} / \mathrm{m}^{3}$, and shear modulus $G=2.59 \times 10^{10}$ $\left.\mathrm{N} / \mathrm{m}^{2}\right) .{ }^{12}$ The outer radius of the solid and hollow waveguides $r_{o}=1.22 \mathrm{~mm}$ and their length $L=306 \mathrm{~mm}$. The inner radius of the hollow waveguide $r_{i}=0.78 \mathrm{~mm}$. The measured speeds of the torsional stress wave in solid and hollow

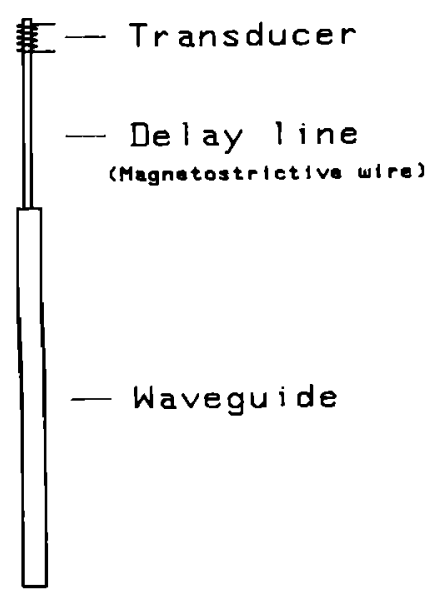

FIG. 1. Schematic description of the torsional wave sensor. waveguides in air at $25^{\circ} \mathrm{C}$ are, respectively, 3014 and 3019 $\mathrm{m} / \mathrm{s}$, which agree within $3 \%$ with the corresponding speed calculated from nominal material properties $\left(G / \rho_{s}\right)^{1 / 2}$. We also report results for a threaded (NF-UNF 3-56) hollow waveguide having similar $r_{o}, r_{i}$, and $L$ to the aforementioned ones. The dominant frequency is estimated as $90 \mathrm{kHz}$. The delay line is made of Remendur (Fe-Co-V-Mn) of length about $1000 \mathrm{~mm}$. The reflectivity of the delay linewaveguide interface can be controlled by controlling the mechanical impedance mismatch at the interface. This is typically done by soldering a small ring around the waveguide.

\section{MATHEMATICAL MODEL}

Consider a torsional stress wave traveling in a uniform circular tube of length $L$, shear modulus $G$, and outer and inner radii $r_{o}$ and $r_{i}$, respectively. The tube is sufficiently long $\left(L / r_{o} \gg 1\right)$ to render end effects negligible. It is submerged in a fluid of viscosity $\mu$. As the torsional wave travels through the waveguide, the solid-liquid interface is alternately accelerated and decelerated. As a result of viscous drag, motion is induced in the fluid.

The circumferential displacement $u_{\theta}$ of the solid (see, for example, Ref. 13) and the circumferential velocity $v_{\theta}$ of the fluid (see, for example, Ref. 14) satisfy, respectively, the conservation equations

$$
\begin{array}{ll}
\frac{\partial^{2} u_{\theta}}{\partial t^{2}}=\frac{\partial^{2} u_{\theta}}{\partial r^{2}}+\frac{1}{r} \frac{\partial u_{\theta}}{\partial r}-\frac{u_{\theta}}{r^{2}}+\frac{\partial^{2} u_{\theta}}{\partial z^{2}}, \quad r_{i} \leqslant r \leqslant 1, \\
\frac{\partial v_{\theta}}{\partial t}=\frac{1}{R}\left(\frac{\partial^{2} v_{\theta}}{\partial r^{2}}+\frac{1}{r} \frac{\partial v_{\theta}}{\partial r}-\frac{v_{\theta}}{r^{2}}+\frac{\partial^{2} v_{\theta}}{\partial z^{2}}\right), \quad 1 \leqslant r<\infty,
\end{array}
$$

with the boundary and interfacial conditions

$$
\begin{aligned}
& \frac{\partial u_{\theta}}{\partial r}-\frac{u_{\theta}}{r}=0, \quad \text { at } r=r_{i}, \\
& \frac{\partial u_{\theta}}{\partial r}-\frac{u_{\theta}}{r}=\frac{\rho}{R}\left(\frac{\partial v_{\theta}}{\partial r}-\frac{v_{\theta}}{r}\right), \quad \text { at } r=1, \\
& v_{\theta}=\frac{\partial u_{\theta}}{\partial t}, \quad \text { at } r=1, \\
& v_{\theta} \rightarrow 0, \quad \text { as } r \rightarrow \infty .
\end{aligned}
$$

Above, all quantities are in nondimensional form. Here, $r$ is the radial distance, and $r_{i}$ is the inner radius of the tube. The length scale is the tube's outer radius $r_{o}$. The velocity scale is $c_{0}=\left(G / \rho_{s}\right)^{1 / 2}$. We shall present our results in terms of the two nondimensional parameters: the density ratio ( $\rho=\rho_{f} / \rho_{s}$ ) and $R=\rho_{f} r_{a} c_{0} / \mu$. Note that $R$ is inversely proportional to the viscosity.

We seek solutions of the form

$$
\begin{aligned}
& u_{\theta}(r, z, t)=U(r) \exp \{-h z+i \omega[(z / c)-t]\}, \\
& v_{\theta}(r, z, t)=V(r) \exp \{-h z+i \omega[(z / c)-t]\},
\end{aligned}
$$

where $\omega, c$, and $h$ are, respectively, the wave's frequency, speed, and attenuation.

In the next section, we shall develop an exact solution for Eqs. (1)-(6), while in Sec. IV, we shall employ a perturbation approach to obtain explicit relations between $c$ and $h$ on the one hand, and $R$ and $\rho$ on the other hand. 


\section{EXACT SOLUTION}

Equations (1) and (2) admit solutions in terms of Bessel functions with complex arguments of the form

$$
U(r)=C_{1} I_{1}\left(A_{s} r\right)+C_{2} K_{1}\left(A_{s} r\right)
$$

and

$$
V(r)=C_{3} I_{1}\left(A_{f} r\right)+C_{4} K_{1}\left(A_{f} r\right),
$$

where $I$ and $K$ are modified Bessel functions of the first and second kind:

$$
A_{s}=\sqrt{(\omega / c+i h)^{2}-\omega^{2}}
$$

and

$$
A_{f}=\sqrt{(\omega / c+i h)^{2}-i \omega R} .
$$

Upon invoking the boundary and interfacial conditions in Eqs. (3)-(6), we obtain the eigenvalue problem for $h$ and c:

$$
\begin{aligned}
F(h, c)= & \frac{1}{i \omega} A_{s}\left(\frac{I_{2}\left(A_{s}\right)}{I_{2}\left(A_{s} r_{i}\right)}-\frac{K_{2}\left(A_{s}\right)}{K_{2}\left(A_{s} r_{i}\right)}\right) \\
& -\frac{\rho}{R} A_{f} \frac{K_{2}\left(A_{f}\right)}{K_{1}\left(A_{f}\right)}\left(\frac{I_{1}\left(A_{s}\right)}{I_{2}\left(A_{s} r_{i}\right)}+\frac{K_{1}\left(A_{s}\right)}{K_{2}\left(A_{s} r_{i}\right)}\right)=0 .
\end{aligned}
$$

When the waveguide is solid $\left(r_{i}=0\right)$, Eq. (13) reduces to

$$
F(h, c)=\frac{1}{i \omega} A_{s} \frac{I_{2}\left(A_{s}\right)}{I_{1}\left(A_{s}\right)}-\frac{\rho}{R} A_{f} \frac{K_{2}\left(A_{f}\right)}{K_{1}\left(A_{f}\right)}=0 .
$$

Equations (13) and (14) are complex equations, and they provide implicit relationships between $h$ and $c$ on the one hand, and $\rho, R, \omega$, and $r$, on the other hand. That is, upon finding the roots of $\mathrm{Eq}$. (13), one can obtain the explicit relations $c=c\left(\rho, R, \omega, r_{i}\right)$ and $h=h\left(\rho, R, \omega, r_{i}\right)$. To this end, we employ Newton's technique. Briefly, we start with an initial guess, $c_{i}$ and $h_{i}$, which may fail to satisfy Eq. (13). Next, we proceed to calculate as correction, $\Delta c$ and $\Delta h$, so as to satisfy Eq. (13). This leads to the complex equation

$F(c+\Delta c, h+\Delta c) \approx F(c, h)+\frac{\partial F}{\partial c} \Delta c+\frac{\partial F}{\partial h} \Delta h=0$.

Upon separation into real and imaginary parts, Eq. (15) yields two algebraic equations that allow one to calculate $\Delta c$ and $\Delta h$. Now, the corrected values $c_{i+1}=c_{i}+\Delta c$ and $h_{i+1}=h_{i}+\Delta h$ play the role of the new initial guesses. The process is repeated until the desired convergence conditions are satisfied. In addition to developing our own routine, we also used, with similar results, the rootfinder function available in Ref. 15. A successful search necessitated, however, a good initial guess. We provided such an initial guess by utilizing the implicit function theorem. Briefly, suppose $c_{i}$ and $h_{i}$ satisfy Eq. (13) for given values of $R, \rho$, and $\omega$. We predict the new values $c_{i+1}=c_{i}+(\partial c / \partial R) \times d R$ and $h_{i+1}=h_{i}$ $+(\partial h / \partial R) \times d R$ corresponding to $R+d R$ by computing the partial derivatives $\partial c / \partial R$ and $\partial h / \partial R$ from the complex equation

$$
\frac{\partial F}{\partial R}+\frac{\partial F}{\partial c} \frac{\partial c}{\partial R}+\frac{\partial F}{\partial h} \frac{\partial h}{\partial R}=0
$$

Once we have calculated the wave speed $c$ and the attenuation $h$, we can proceed and calculate the group velocity

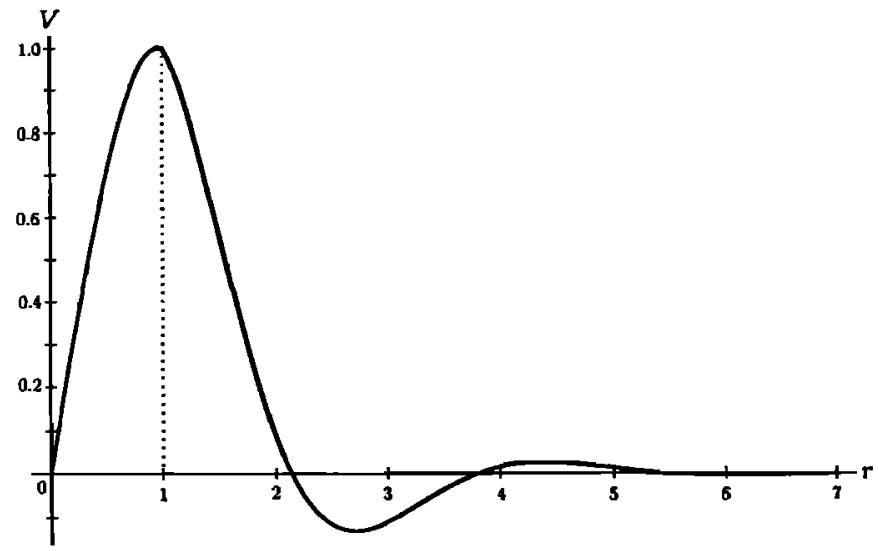

FIG. 2. Velocity profile of the solid and the fluid when $\omega=1.0, \rho=0.1$, and $R=0.2$. The vertical dashed line denotes the location of the solid-fluid interface.

$c_{g}=d \omega / d k$ by differentiating Eq. (13) with respect to the wave number $k$.

A sample of our results for a solid cylinder and for the fundamental torsional mode is depicted in Figs. 2-4. Additional results are available in Ref. 16. In Fig. 2, we depict the instantaneous velocity field in the solid $\left(\partial u_{\theta} / \partial t\right)$ and the surrounding fluid $\left(v_{\theta}\right)$ as functions of the radial distance $(r)$ from the waveguide's center. The vertical dashed line in Fig. 2 denotes the location of the solid-fluid interface. The velocity field is shown for a relatively high-viscosity liquid $(R=0.2)$. Thus we have a relatively thick boundary layer in the fluid.

In Fig. 3(a) and (b), we use symbols to depict the dependence of the attenuation [Fig. 3(a)] and the phase velocity [Fig. 3(b)] on the fluid's viscosity $(R)$ for three density ratios $\rho=0.1,1.0$, and 5.0. The solid lines in Fig. 3 correspond to asymptotic solutions which we shall describe in the next section. In the range of $R$ values considered, as expected, the attenuation [Fig. 3(a)] increases monotonically with decreasing $R$. In other words, as the fluid viscosity increases, so does the damping. The same is not true for the phase velocity. For relatively large $R$ values, the phase speed decreases as $R$ decreases [Fig. 3(b) ]. This is expected, since as $R$ decreases, the thickness of the fluid's boundary layer increases (i.e., a larger mass of fluid is engaged in the motion) which, in turn, implies that the stress wave cncounters larger inertia and thus there is a slowdown in speed. As $R$ is further decreased, we observe that the phase speed attains a minima and then increases again. In order to explain this change in trend, it is useful to examine the effect of $R$ on the shape of the displacement field in the solid. To this end, we depict in Fig. 4, the displacement $u_{\theta}$ in the solid as a function of the radial distance $r$ for various $R$ values. For comparison purposes, all the curves in Fig. 4 have the same slope at the origin. For relatively large values of $R$ (i.e., $R>10$ ), the displacement (Fig. 4) is almost proportional to the radial dis- 


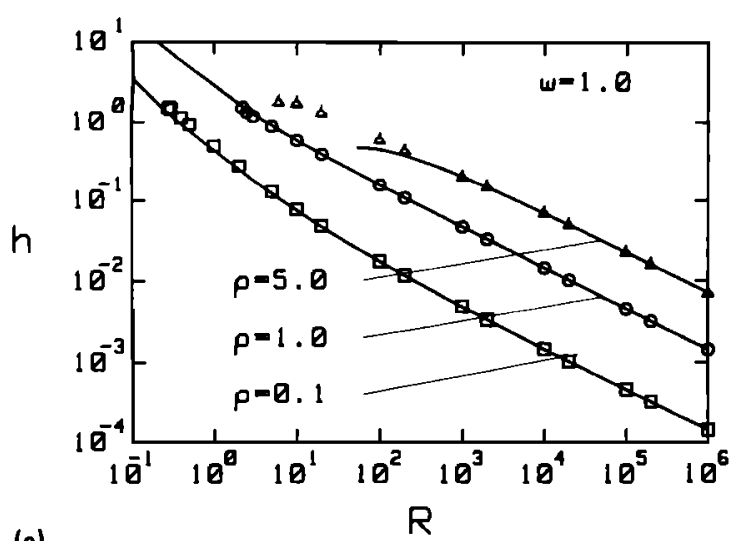

(a)

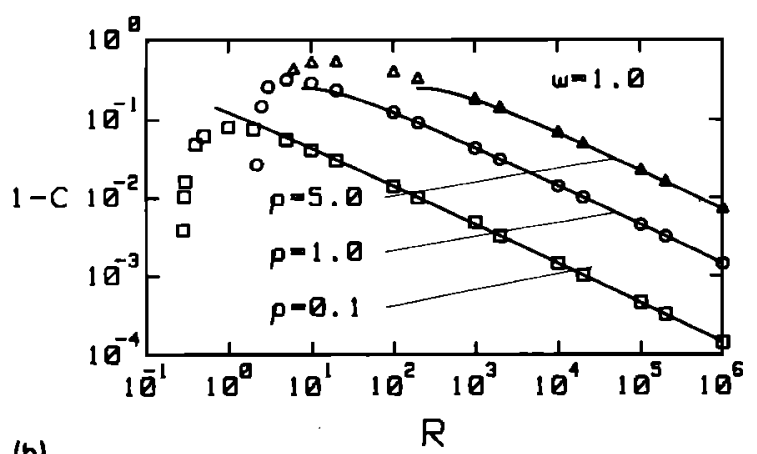

(b)

FIG. 3. (a) The attenuation $h$ depicted as a function of $R$ for $\omega=1.0$ and various $\rho$. The solid lines denote the asymptotic solutions while the symbols denote the exact solution. (b) The phase speed $c$ depicted as a function of $R$ for $\omega=1.0$ and various $\rho$. The solid lines denote the asymptotic solutions while the symbols denote the exact solution.

tance resembling the fundamental mode in the classical torsion problem. ${ }^{17}$ As $R$ decreases, the displacement curve starts bending until, eventually, a nodal point is attained. The displacement field for smaller values of $R$ (i.e., $R \leqslant 0.2$ ) resembles somewhat the second torsional mode in the classical torsion problem. Since the second torsional mode has a higher phase speed than the fundamental one, this explains the increase in the wave speed as $R$ decreases below a certain value (Fig. 4).

We also calculated the group velocity (the results are not shown here, for details see Ref. 18) and found it to be slightly higher than the phase velocity.

\section{ASYMPTOTIC SOLUTION FOR LARGE $\boldsymbol{R}$}

The exact procedure developed in Sec. III suffers from the disadvantage that $c$ and $h$ are not expressed explicitly in terms of the parameters $R$ and $\rho$. In many applications, however, $R$ is fairly large. For example, for a circular waveguide of outer radius $r_{o}=1.22 \mathrm{~mm}$ submerged in glycerin at room temperature, $R$ is 2270 . Thus it may be useful to obtain an asymptotic solution for large $R$. To this end, we introduce the parameter $\epsilon=R^{-1 / 2}$ and employ for the fluid the stretched coordinate $\eta=(r-1) / \epsilon$. Upon rescaling, the governing Eqs. (1)-(6) accept the form

$$
\begin{aligned}
& U^{\prime \prime}(r)+(1 / r) U^{\prime}(r) \\
& \quad+\left[\omega^{2}+\left(-h+i \frac{\omega}{c}\right)^{2}-\frac{1}{r^{2}}\right] U(r)=0, \quad r_{i} \leqslant r \leqslant 1 \\
& V^{\prime \prime}(\eta)+\frac{\epsilon}{1+\epsilon \eta} V^{\prime}(\eta)+\left[i \omega+\left(-h+i \frac{\omega}{c}\right)^{2} \epsilon^{2}\right. \\
& \left.\quad-\frac{\epsilon^{2}}{(1+\epsilon \eta)^{2}}\right] V(\eta)=0, \quad 0 \leqslant \eta<\infty \\
& U^{\prime}(r)-(1 / r) U(r)=0 \quad \text { at } r=r_{i}, \\
& U^{\prime}(r)-\frac{1}{r} U(r)=\rho\left(\epsilon V^{\prime}(\eta)-\frac{\epsilon^{2}}{1+\epsilon \eta} V(\eta)\right) \\
& \quad \text { at } r=1 \quad \text { and } \eta=0, \\
& V(\eta)=-i \omega U(r) \quad \text { at } r=0 \text { and } \eta=0 \\
& V(\eta) \rightarrow 0 \text { as } \eta \rightarrow \infty \text {. }
\end{aligned}
$$

The case of $R \rightarrow \infty(\epsilon \rightarrow 0)$ corresponds to the classical torsional problem in a circular cylinder submerged in inviscid fluid. In what follows, we shall develop a solution that, in the limit of $R \rightarrow \infty$, reduces to the fundamental mode of the classical case. A similar procedure may be used for other modes.

We seek series solutions in the form

$$
\begin{aligned}
& U(r)=\bar{U}_{0} r+\sum_{n=1}^{\infty} \epsilon^{n} u_{n}(r), \\
& V(\eta)=\sum_{n=0}^{\infty} \epsilon^{n} v_{n}(\eta), \\
& c=1+\sum_{n=1}^{\infty} \epsilon^{n} c_{n}, \\
& h=\sum_{n=1}^{\infty} \epsilon^{n} h_{n} .
\end{aligned}
$$

Next, we substitute the series of Eqs. (23)-(26) into Eqs. (17)-(22) and equate coefficients of like powers in $\epsilon$. As a result, we obtain a sequence of boundary value problems which can be solved recursively to obtain the explicit expressions for $c$ and $h$ and the group velocity $c_{g}$ in the form

$$
\begin{aligned}
c=1 & -\frac{1}{\sqrt{R}} \frac{1}{1-r_{i}^{4}} \rho \sqrt{\frac{2}{\omega}}+\frac{1}{R} \frac{1}{\left(1-r_{i}^{4}\right)^{2}} \frac{2 \rho^{2}}{\omega} \\
& +O\left(\frac{1}{(\sqrt{R})^{3}}\right)
\end{aligned}
$$

$$
\begin{aligned}
h= & \frac{1}{\sqrt{R}} \frac{1}{1-r_{i}^{4}} \rho \sqrt{2 \omega}+\frac{1}{R}\left(-2 \frac{r_{i}^{4}\left(1-r_{i}^{2}\right)}{\left(1-r_{i}^{4}\right)^{3}} \rho^{2} \omega^{2}\right. \\
& -\frac{2}{3} \frac{1-r_{i}^{6}}{\left(1-r_{i}^{4}\right)^{3}} \rho^{2} \omega^{2}+\frac{1+r_{i}^{4}}{\left(1-r_{i}^{4}\right)^{2}} \rho^{2} \omega^{2} \\
& \left.-\frac{2}{\left(1-r_{i}^{4}\right)^{2}} \rho^{2}+\frac{3}{1-r_{i}^{4}} \rho\right)+O\left(\frac{1}{(\sqrt{R})^{3}}\right),
\end{aligned}
$$




$$
\begin{aligned}
c_{g}= & -\frac{1}{\sqrt{R}} \frac{1}{1-r_{i}^{4}} \frac{\rho}{\sqrt{2 \omega}}+\frac{1}{R} \frac{1}{\left(1-r_{i}^{4}\right)^{2}} \frac{\rho^{2}}{2 \omega} \\
& +o\left(\frac{1}{(\sqrt{R})^{3}}\right) .
\end{aligned}
$$

In principle, the procedure could be continued indefinitely. Due to the increasing length of the expressions involved, we calculated only the first three terms in the series. Since Eqs. (27)-(29) represent a truncated Taylor series, they are likely to be valid only for a limited range of $R$ values. We can estimate the range of validity of the asymptotic series by utilizing only the first two terms in the series, and using the third term as an estimate of the truncation error. Alternatively, the range of validity can be determined by comparing the approximate results of Eqs. (27)-(29) with the exact ones (Sec. III). To this end, we depict the results of the perturbation analysis as solid lines in Fig. 3. It is clear from Fig. 3 that for a wide range of $R$ values, the approximate solution is in excellent agreement with the exact one. In Table $I$, we list the smallest $R$ value as a function of the wave number $k$ and the density ratio $\rho$, above which the deviation of the asymptotic solution from the exact one is smaller than $1 \%$.

We note in passing that the $O(\epsilon)$ terms in Eqs. (27)(29) are identical to what one obtains when one assumes that the fluid's drag does not affect the deformation field in the solid. That is, the flow field in the fluid is identical to the one generated by a flat, infinite plate submerged in viscous fluid and oscillating in its own plane. ${ }^{19}$ For large $R$, we can neglect the curvature of the circular cross section due to the extremely small thickness of the viscous boundary layer. The higher-order terms reflect the effect of the viscous drag on the deformation field. Figure 4 shows that this effect becomes significant only for relatively small values of $R$. This explains why the approximate technique is so successful over a wide range of $R$ values.

\section{COMPARISON WITH EXPERIMENTS}

In our experiments, we measured the time ( $D t)$ elapsing between reflections from the delay line-waveguide interface and the waveguide's end, as well as the corresponding amplitudes of the reflected signals, which we denoted as $A$ and $B$, respectively. Since the length of the waveguide is known, we can readily calculate the speed of the wave. The standard deviation for the speed measurement is smaller than $0.2 \%$. The attenuation can be computed from our knowledge of amplitudes $A$ and $B$. However, to perform such a calcula-

TABLE I. Approximate smallest values of $R$ for various wave number $k$ and density ratio $\rho$, below which the deviation of the asymptotic solution from the exact numerical results becomes larger than $1.0 \%$.

\begin{tabular}{crrr}
\hline \hline & & $R$ & \\
$\rho$ & $k=0.1$ & $k=1.0$ & $k=10.0$ \\
\hline 0.1 & 45.0 & 3.0 & 8.0 \\
1.0 & 100.0 & 30.0 & 80.0 \\
5.0 & 10000.0 & 700.0 & 750.0 \\
\hline \hline
\end{tabular}

tion, knowledge of the reflection coefficient $C_{R}$ at the delay line-waveguide interface is necessary. We assume that, to the first approximation, the reflection coefficient is independent of the fluid's viscosity, and obtain its value from measurements conducted while the waveguide is submerged in air (attenuation is assumed to be negligibly small). Thus we find $^{20}$

$$
C_{R}=\frac{1}{2}\left[-\frac{B}{A}+\sqrt{\left(\frac{B}{A}\right)^{2}+4}\right] .
$$

Once $C_{R}$ is known, we can calculate the nondimensional attenuation $h$ from the equation

$$
h=\frac{r_{o}}{2 L} \log \left(\frac{A}{B} \frac{1-C_{R}^{2}}{C_{R}}\right) .
$$

The standard deviation for the measured attenuation is estimated to be smaller than $1 \%$.

We carried out our experiments with Cannon viscosity standard liquids: $\mathrm{S} 6, \mathrm{~S} 60, \mathrm{~S} 600$, and $\mathrm{N} 100^{21}$ at temperatures $20,25,40$, and $50^{\circ} \mathrm{C}$. The range of viscosities and densities covered was $7-2000 \times 10^{-3} \mathrm{~kg} / \mathrm{m}$ s and $0.8-1.2 \times 10^{3}$ $\mathrm{kg} / \mathrm{m}^{3}$, respectively. The corresponding ranges of $R$ and $\rho$ values were $2270 \sim 432000$ and $0.28 \sim 0.44$. The standard liquids were contained in a constant, uniform (within $0.5^{\circ} \mathrm{C}$ ) temperature chamber.

The theoretically predicted and measured $h$ and $c$ are shown in Figs. 5 and 6 as functions of the product $\left(\rho_{f} \mu\right)^{1 / 2}$. Experimental results are shown for smooth solid waveguides (solid circles); smooth hollow waveguides (open circles with a central dot); and threaded hollow waveguides (open circles). The dashed lines represent a linear least-squares fit through the experimental points. Theoretical results are given only for the smooth solid (solid line) and smooth hollow (dash-dot line) waveguides. For the range of $R$ values considered, both the experimental and theory show essentially linear dependence between the wave speed and the attenu-

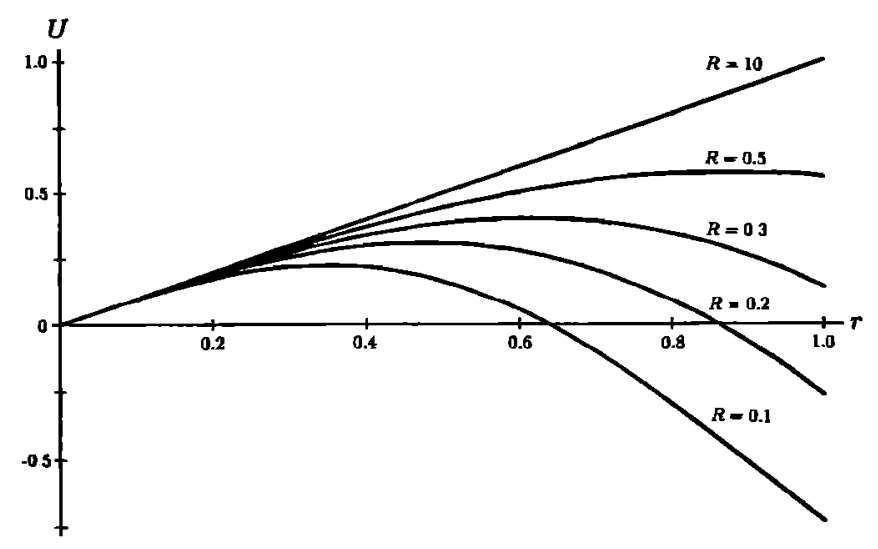

FIG. 4. The deformation field associated with the fundamental mode depicted as a function of the radial distance $r$ for various $R$ when $\omega=1.0$ and $\rho=0.1$. 


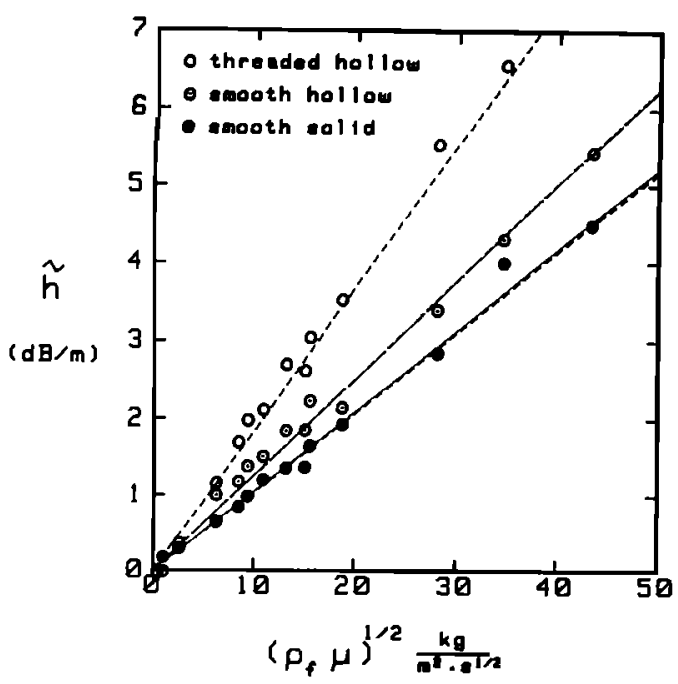

FIG. 5. The attenuation coefficient displayed as a function of $\left(\rho_{f} \mu\right)^{1 / 2}$. The solid lines represent the theoretical solution. The circles and the dashed lines represent the experimental results and the linear least-squares fit obtained from these results.

ation on the one hand and $\left(\rho_{f} \mu\right)^{1 / 2}$ on the other hand. The agreement between the predicted and the measured attenuation $(h)$ in Fig. 5 is better than $1 \%$.

The agreement between the predicted wave speed and the measured one in Fig. 6 is not always as good. For the solid waveguide, we obtain good agreement between the experiment and theory for $\left(\rho_{j} \mu\right)^{1 / 2}<20$. But, for $\left(\rho_{f} \mu\right)^{1 / 2}>20$, there is about a $10 \%$ discrepancy between experiment and theory. For the hollow waveguide, the discrepancy between theory and experiment is even larger (about a factor of 1.5 ). In the next section, we shall discuss a few possible reasons for these discrepancies.

\section{CONCLUSIONS AND DISCUSSION}

In this paper, we considered theoretically and experimentally the effect of an adjacent fluid's viscosity on the characteristics of a torsional stress wave transmitted in a waveguide with a circular cross section. The results may be useful for the design of a viscosimeter. The advantage of the sensor described here is that it can be positioned permanently in line to provide real time viscosity measurement.

The theory developed here allows one to correlate a stress wave's wave speed and attenuation with the adjacent fluid's characteristics. The theory is more rigorous than any heretofore available since it accounts for the fluid effect on the deformation field in the solid. The theory is not convenient to use since it requires the solution of transcendental equations and does not lead to an explicit relationship between the quantities of interest. This shortcoming is partially alleviated by developing asymptotic expressions for the wave's speed and attenuation. The approximate expressions are in good agreement with the exact ones for a wide range of parameter values.
The theoretical results were compared with experimental observation. For the wave attenuation, we obtained a favorable agreement between theory and experiment (Fig. 5).

For the wave speed (Fig. 6) in solid waveguides submerged in relatively low viscosity liquids $\left(\rho_{f} \mu\right)^{1 / 2}<20$, we observe good agreement between the experiment and theory. For solid waveguides submerged in high viscosity liquids $\left[\left(\rho_{f} \mu\right)^{1 / 2}>20\right]$ and hollow waveguides submerged in any liquid, the experimental results deviate significantly from the theoretical predictions. The measured speed is considerably slower than the predicted one. We do not have a complete explanation for this discrepancy. Below, we briefly discuss a few possible reasons. The fact that the observed speed is lower than the predicted one implies that the theory may be underestimating the apparent inertia of the fluid. Such an underestimation could occur if secondary fluid motion (which was not included in our theory) were present. Indeed, researchers have observed secondary flows in the case of oscillating cylinders ${ }^{22}$ when the Taylor number $T=2 u_{\theta}^{2}(2 \omega)^{1 / 2} /\left(r_{0} v^{1 / 2}\right)>164$. However, such secondary flows are not likely to be present in our experiments as typically the Taylor number $T \ll 1$. Another possible reason for the discrepancy is that the theory assumes an essentially monochromatic wave while, in actuality, we are dealing with a broadband of frequencies. Also, especially in the case of the hollow cylinder, it is possible that in addition to the torsional oscillations other modes of vibrations such as angular Lamb waves are included. The simple theory we have presented here does not account for this possibility. Clearly, in the case of the hollow waveguide, there is a need to develop a more complete theory than the one presented here. In any event, given these discrepancies, if viscosity were to be inferred from wave speed, it would be necessary to calibrate the sensor rather than rely on the theoretical correlation. Given the linear relationship between the wave speed and $\left(\rho_{f} \mu\right)^{1 / 2}$, this should not be a formidable task.

In this work we showed that a torsional wave sensor can be used to measure the $\left(\rho_{f} u\right)^{1 / 2}$ of Newtonian fluids. Thus one can obtain a fluid's viscosity if its density is known. Often, it is desired to measure both density and viscosity independently. In theory, one could obtain both the viscosity and density from the attenuation and speed measurements. However, Eqs. (27)-(29) suggest that the functional difference in the effect of viscosity and density on wave speed and attenuation is a second-order effect. Thus it may not be practical to obtain both $\rho$ and $\mu$ from a single sensor. If the measurement of both density and viscosity is required, one might consider transmitting torsional stress waves in two waveguides: one with a circular cross section and the other with a noncircular cross section (see, for example, Refs. 4 and 23).

Finally, an issue of considerable interest is how to improve the sensor's sensitivity. In this paper, we report results pertaining to solid and hollow waveguides. Clearly, hollow waveguides outperform their solid counterparts (Figs. 5 and 6). The thinner the wall thickness, the better. For example, if we measure wave speed/attenuation, the hollow waveguide used by us is $70 \% / 20 \%$ more sensitive than the solid one. Additional gains in sensitivity can be obtained by increasing the surface area of the waveguide in contact with the fluid so 
as to increase the fluid's apparent inertia. This can be accomplished by corrugating or threading the waveguide's surface. For example, the threaded hollow waveguide we used improved sensitivity for speed/attenuation measurements by an additional $60 \% / 50 \%$ (Figs. 5 and 6 ) compared with the smooth hollow waveguide. Further increases in sensitivity are possible by using external confinement. For example, the viscous fluid could be enclosed between the waveguide and an external coaxial tube. Indeed, such a confinement may be desired to provide a mechanical protection for the waveguide. We report some theoretical results on the effect of external confinement in the Appendix. Here, we note only that for such an arrangement to be effective, the gap between the external tube and the waveguide should be pretty small, typically less than $10 \%$ of the waveguide's radius. Such a small gap may be feasible only in applications involving relatively clean fluids.

\section{ACKNOWLEDGMENTS}

This work has been supported by the National Science Foundation (Grant No. CBT 83-51 658), Panametrics, Inc., and the Electric Power Research Institute (RP-251503 ). We gratefully acknowledge useful discussions with $\mathrm{L}$. C. Lynnworth and T. H. Nguyen of Panametrics Inc.

\section{APPENDIX: THE EFFECT OF EXTERNAL CONFINEMENT ON THE SPEED AND ATTENUATION OF TORSIONAL STRESS WAVES}

In this Appendix, we consider the effect of external confinement on the sensor's performance. Our objectives are twofold. First, since external confinement may be employed in applications to provide a mechanical protection for the sensor, it is desired to establish whether such a protection will affect the sensor's performance. Second, we wish to examine if the sensor's sensitivity can be increased by deploying such a confinement.

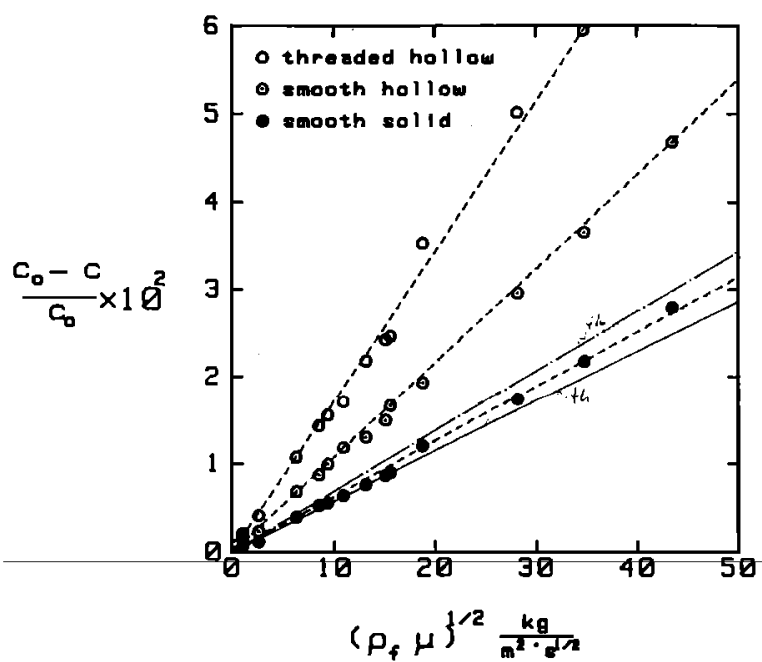

FIG. 6. The wave speed displayed as a function of $\left(\rho_{f} \mu\right)^{1 / 2}$. The solid lines represent the theoretical solution. The circles and the dashed lines represent the experimental results and the linear least-squares fit obtained from these results.

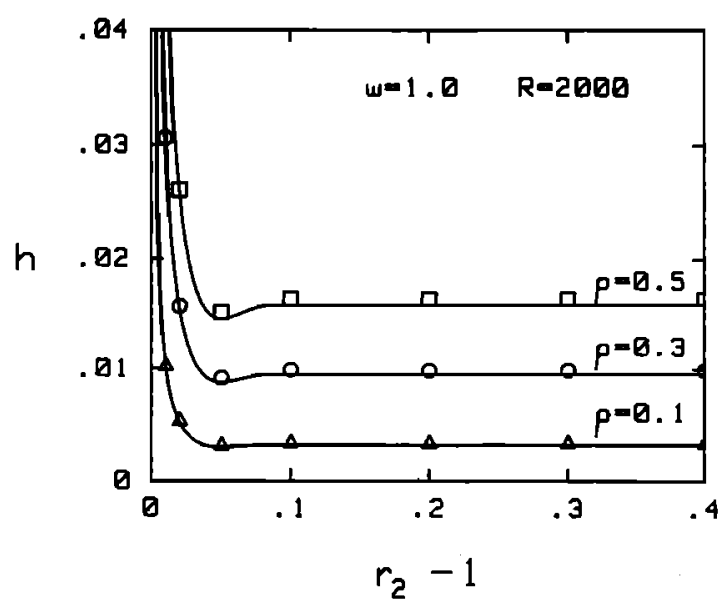

FIG. Al. The attenuation $h$ depicted as a function of gap width for various $\rho$ when $\omega=1.0$ and $R=2000$. The solid lines denote the asymptotic solutions, while the symbols denote the exact solution.

We shall report here both exact and asymptotic results. For brevity's sake, we consider only the case of the smooth solid waveguide confined inside a concentric external tube of inner radius $r_{2}$. To the first approximation, we assume that the external tube is rigid. We solve Eqs. (1)-(5) with Eq. (6) being replaced by the nonslip condition

$$
v_{\theta}=0 \text {, at } r=r_{2} \text {. }
$$

The resulting eigenvalue problem for the wave speed $(c)$ and attenuation $(h)$ is

$$
\begin{gathered}
F(h, c)=\frac{1}{i \omega} A_{s} \frac{I_{2}\left(A_{s}\right)}{I_{1}\left(A_{s}\right)}\left(-\frac{I_{2}\left(A_{f}\right)}{I_{1}\left(A_{f} r_{2}\right)}+\frac{K_{1}\left(A_{f}\right)}{K_{1}\left(A_{f} r_{2}\right)}\right) \\
-\frac{\rho}{R} A_{f}\left(\frac{I_{2}\left(A_{f}\right)}{I_{1}\left(A_{f} r_{2}\right)}+\frac{K_{2}\left(A_{f}\right)}{K_{1}\left(A_{f} r_{2}\right)}\right)=0,
\end{gathered}
$$

where the significance of the various variables is given in the body of the paper. The corresponding asymptotic expressions for the wave speed and attenuation are

$$
\begin{aligned}
c=1 & -\frac{1}{\sqrt{R}} \rho \sqrt{\frac{2}{\omega}} \frac{\sinh \left(\sqrt{2 \omega} \eta_{2}\right)-\sin \left(\sqrt{2 \omega} \eta_{2}\right)}{\cosh \left(\sqrt{2 \omega} \eta_{2}\right)-\cos \left(\sqrt{2 \omega} \eta_{2}\right)} \\
& +O\left(\frac{1}{R}\right), \\
h= & \frac{1}{\sqrt{R}} \rho \sqrt{2 \omega} \frac{\sinh \left(\sqrt{2 \omega} \eta_{2}\right)+\sin \left(\sqrt{2 \omega} \eta_{2}\right)}{\cosh \left(\sqrt{2 \omega} \eta_{2}\right)-\cos \left(\sqrt{2 \omega} \eta_{2}\right)}+O\left(\frac{1}{R}\right),
\end{aligned}
$$

where $\eta_{2}=\sqrt{R}\left(r_{2}-1\right)$. Note that for $r_{2} \rightarrow \infty$, the expressions in Eqs. (A2) - (A4) reduce to the case of a solid waveguide embedded in an infinite fluid.

The attenuation and the wave speed are depicted as functions of the gap width $\left(r_{2}-1\right)$ in Figs. A1 and A2 for various $\rho$ when $R=2000, \rho=0.3$, and $\omega=1$. The solid lines and the symbols correspond to the asymptotic and the exact solutions, respectively. The figures demonstrate that if 


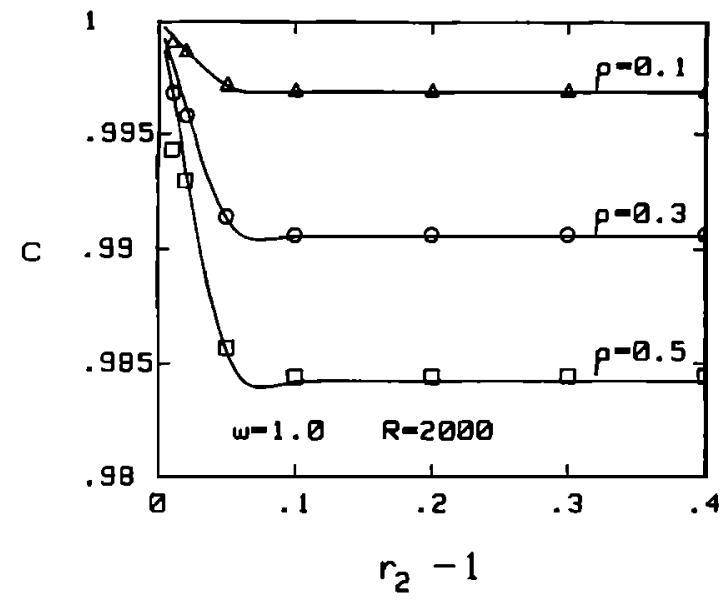

FIG. A2. The wave speed $c$ depicted as a function of gap width for various $\rho$ when $\omega=1.0$ and $R=2000$. The solid lines denote the asymptotic solutions while the symbols denote the exact solution.

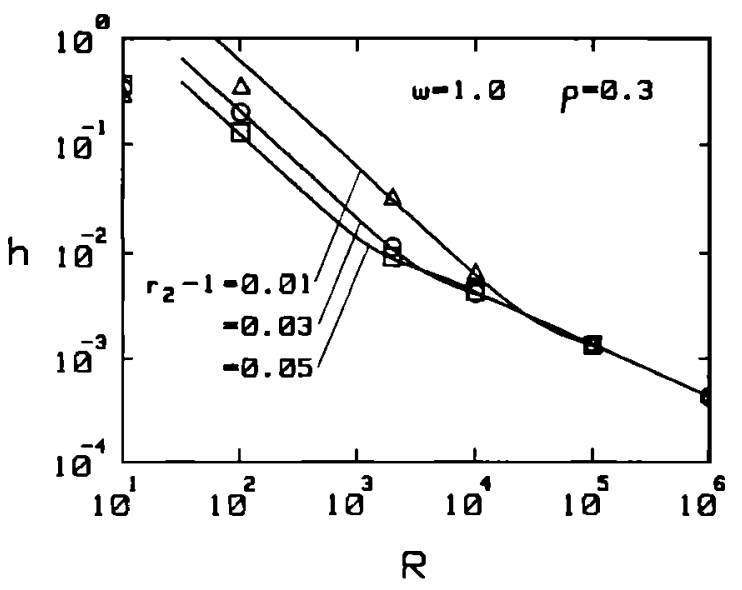

FIG. A3. The attenuation $h$ depicled as a function of $R$ for various gap width when $\omega=1.0$ and $\rho=0.3$. The solid lines denote the asymptotic solutions while the symbols denote the exact solution.

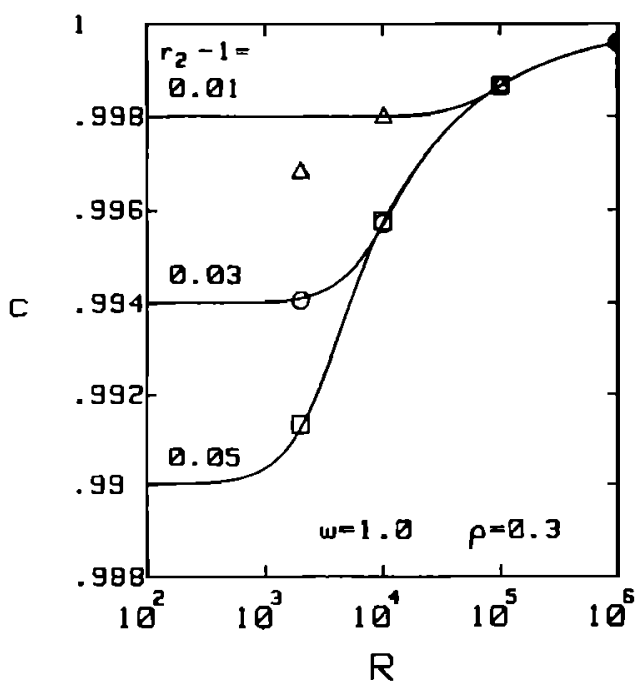

FIG. A4. The wave speed $c$ depicted as a function of $R$ for various gap width when $\omega=1.0$ and $\rho=0.3$. The solid lines denote the asymptotic solutions, while the symbols denote the exact solution. the gap thickness is larger than $10 \%$ of the waveguide's radius, the results are essentially identical to those of the unconfined case. Under this circumstance, the thickness of the fluid's boundary layer is smaller than the gap thickness and the external confinement has little or no effect. As the gap thickness decreases, the speed and the attenuation decrease slightly to obtain a minimum around $r_{2}-1=0.05$. A further decrease in the gap width causes an increase in the wave speed and attenuation. Thus the effects of external confinement are felt only at small gap thicknesses. The aforementioned values for gap thickness depend on the fluid's characteristics and are likely to increase as the fluid viscosity increases.

Next, we examine the effect of gap thickness on the sensor's sensitivity. To this end, we depict in Figs. A3 and A4 the wave speed and attenuation as functions of $R$ for various gap thicknesses when $\omega=1.0$ and $\rho=0.3$. Note that the approximation in Eqs. (A3) and (A4) adequately describes the behavior of $c$ and $h$ as functions of $R$ as long as the gap thickness is bigger than the thickness of the viscous boundary layer. That is, $r_{2}-1>(\omega R)^{-1 / 2}$. For smaller gap thicknesses, the asymptotic approximation is no longer valid. Within the range of validity of the asymptotic approximation ( $R>5 \times 10^{2}$ in Figs. A3 and A4), Eqs. (A3) and (A4) suggest, for small $\eta_{2}$, that $h \sim R^{-1}$, while in the unconfined case $h \sim R^{-1 / 2}$ [Eq. (29)]. Thus the sensor's sensitivity can be improved by closely confining the waveguide in an external tube.

'W. Roth and S. R. Rich, "A New Method for Continuous Viscosity Measurement. General Theory of the Ultra-Viscoson," J. Appl. Phys. 24(7), 940-950 (1953).

${ }^{2}$ L. C. Lynnworth, "New Design for Magnetostrictive Probes Using Extensional, Torsional, and Flexural Waves," in Ultrasonics Symposium Proceedings (IEEE, New York, 1978), pp. 300-304; also in Ultrasonic Measurements for Process Control (Academic, New York, 1989).

J. O. Kim and H. H. Bau, "On Line, Real-Time Densiometer-Theory and Optimization," J. Acoust. Soc. Am. 85, 432-439 (1989).

4J. O. Kim and H. H. Bau, "Instrument for Simultaneous Measurement of Density and Viscosity," Rev. Sci. Instrum. 60(6), 1111-1115 (1989).

${ }^{5} \mathrm{~J}$. C. Nieuwoudt, J. Kestin, and J. V. Senger, "On the Theory of Oscillating-Body Viscometer," Physica 142A, 53-74 (1987).

'A. H. Krall, J. C. Nieuwoudt, J. V. Sengers, and J. Kestin, "Feasibility of Simultaneous Viscosity and Density Measurement of a Fluid From the Motion of an Oscillating Disk," Fluid Phase Equilibria 36, 207-218 (1987).

${ }^{\prime} \mathbf{R}$. N. Kleiman, "Analysis of the Oscillating-Cup Viscometer for the Measurement of Viscoelastic Properties," Phys. Rev. A 35, 262-275 (1987).

"J. C. Nieuwoudt, J. V. Sengers, and J. Kestin, "On the Theory of Oscillating-Cup Viscometer," Physica 149A, 107-122 (1988).

'J. V. Fitzgerald and F. J. Matusik, "Oscillation Viscometer," Meas. Control 20(3), 175-179 (1986).

${ }^{10} \mathrm{~K}$. Torklep and H. A. Øye, "An Absolute Oscillating-Cylinder (or Cup) Viscometer for High Temperature," J. Phys. E: Sci. Instrum. 12, 875-885 (1979).

"N. S. Tzannes, "Joule and Wiedemann Effects-The Simultaneous Generation of Longitudinal and Torsional Stress Pulses in Magnetostrictive Materials," lEEE Trans. Sonics and Ultrasonics SU-13, 33-4l (1966).

${ }^{12}$ R. E. Bolz and G. L. Tuve (eds.), CRC Handbook of Tables for Applied Engineering Science, (CRC, Cleveland, 1973), 2nd ed., p. 117.

${ }^{13}$ H. Kolsky, Stress Waves in Solids (Dover, New York, 1963), pp. 65-68.

${ }^{14} \mathrm{H}$. F. Winny, "Rotary Oscillation of a Long Circular Cylinder in a Viscous Fluid," Phil. Mag., Ser. 7 14, 1027-1032 (1932). 
${ }^{15} \mathrm{~S}$. Wolfram, Mathematica-A System for Doing Mathematics by Computer (Addison-Wesley, New York, 1988).

${ }^{16} \mathrm{Y}$. Wang, "The Effects of the Adjacent Viscous Fluid on the Transmission of Torsional Stress Waves in a Solid Waveguide," MS thesis, University of Pennsylvania (1987).

${ }^{17}$ A. E. H. Love, Mathematical Theory of Elasticity (Dover, New York, 1944), pp. 287-289.

${ }^{18}$ J. O. Kim, "The Interaction between Stress Waves in Solid Waveguides and the Adjacent Media," Ph.D. thesis, University of Pennsylvania, 1989.

${ }^{19}$ C.-S. Yih, Fluid Mechanics (West River, Ann Arbor, 1977), pp. 321-324.
${ }^{20}$ L. C. Lynnworth, "Attenuation Measurements Using the Pulse-Echo $A B$ Method, without Multiple Echo Reverberations in Specimen," Materials Eval., 6-9 (1973).

${ }^{21}$ J. F. Swindells, R. C. Hardy, and R. L. Cottington, "Precise Measurements with Bingham Viscometers and Cannon Master Viscometers," J. Res. Natl. Bur. Stand. RP 2479, 52(3), 105-115 (1953).

${ }^{22}$ G. Seminara and P. Hall, "The Centrifugal Instability of a Stokes Layer: Nonlinear theory," Proc. R. Soc. Lond. Ser. A 354, 119-126 (1977).

${ }^{23}$ H. H. Bau, J. O. Kim, L. C. Lynnworth, and T. H. Nguyen, "Improved Torsional Wave Sensor and System," U.S. Pat. 4,893,496, Jan. 16, 1990. 